\title{
THE TIME DIFFERENCE IN EMISSION OF LIGHT AND PRESSURE PULSES FROM OSCILLATING BUBBLES
}

\author{
KAREL VOKURKA \\ Physics Department, Technical University of Liberec, Studentská 2, 46117 Liberec, Czech Republic \\ correspondence: karel.vokurka@tul.cz
}

\begin{abstract}
Oscillations of spark-generated bubbles are studied experimentally. In this work, an attention is paid to the time difference in the radiation of light flashes and pressure pulses from a bubble at the final stages of the first bubble contraction and the early stages of the first bubble expansion. It is found that light and pressure pulses are not radiated synchronously. In some experiments, the light flashes are radiated before the pressure pulses by a few 1 s and in other experiments, the light flashes are radiated later than the pressure pulses by a few $\mu s$. The time difference in the radiation of the two pulses is examined in detail in relation with the bubble size, bubble oscillation intensity, maximum value of the light flash and the width of the light flash. It is shown that the magnitude of the time differences is very weakly correlated with the bubble size, intensity of oscillation and intensity of the light flashes and that the magnitude of the time differences is only moderately correlated with the light flashes widths.
\end{abstract}

KEYWORDS: spark-generated bubbles; light emission; bubble oscillations.

\section{INTRODUCTION}

Bubble oscillations have long been an important topic in fluid dynamics. While they have traditionally been studied in connection with erosion damage [1], recent efforts have been aimed at medical applications such as contrast-enhancing in ultrasonic imaging [27. Physical processes in oscillating bubbles are very complex and so far many points in this field have not yet been clarified. One of these points that is not well understood is the emission of light from bubbles. And this phenomenon will be discussed in this work.

In experiments, oscillating bubbles are generated using a wide variety of techniques. These techniques encompass, e.g. laser beam focusing in the liquid [8] 10], spark discharge in liquid [11-15], multiple bubbles oscillating in ultrasonic fields [16, hydrodynamic cavitation in the liquid flow [17, [18, and shock induced bubble oscillations [19]. All these techniques are also used in studies of the light emission from bubbles [9, 11, 13, 19.

During the last thirty years, the light emission from oscillating bubbles has also been intensively studied in a number of laboratories using acoustic resonators. A recent review of papers published in this area [20] mentions 309 references. Although this review concentrates on the light emission from bubbles oscillating in acoustic resonators, it also includes papers dealing with the light emission from bubbles generated during acoustic cavitation. Papers dealing with the light emission from bubbles generated during hydrodynamic cavitation and from laser- and spark-generated bubbles are not included in this review.

In this work, the emission of light from large sparkgenerated bubbles freely oscillating in water far from boundaries is studied. An obvious advantage of large bubbles is that the optical and acoustic radiation from them can be recorded more easily than in the case of smaller bubbles. This is because, in large oscillating bubbles, physical processes are taking place more slowly. The light emitted by large bubbles is also sufficiently intensive so that averaging on light pulses from different experiments, which is always accompanied by a loss of natural variety (e.g., in pulse shape), is not necessary. The technique of low voltage spark discharges makes it also possible to generate bubbles of different sizes and oscillating with different intensities [21, which further enhances the data analysis.

In this work, the time difference in radiation of the optical and acoustic pulses at the first bubble contraction and at the following first bubble expansion is studied. It will be shown that the instants, when the maxima in the optical pulses and pressure pulses are radiated, may differ by a few ps. In some experiments, the maxima in optical pulses are radiated earlier than the peaks in the pressure pulses, and in some experiments, the maxima of the optical pulses are radiated later than the pressure peaks. This phenomenon has also been observed by Golubnichiy et al. 11, Huang et al. 13] and Zhang et al. 14]. Results discussed here have been presented in a brief form at conferences [22, 23].

\section{EXPERIMENTAL SETUP}

The experimental setup used in this work is schematically shown in Figure 1 Freely oscillating bubbles were generated by discharging a capacitor bank via a sparker submerged in a laboratory water tank having dimensions of $6 \mathrm{~m}$ (length) $\times 4 \mathrm{~m}$ (width) $\times 5.5 \mathrm{~m}$ 


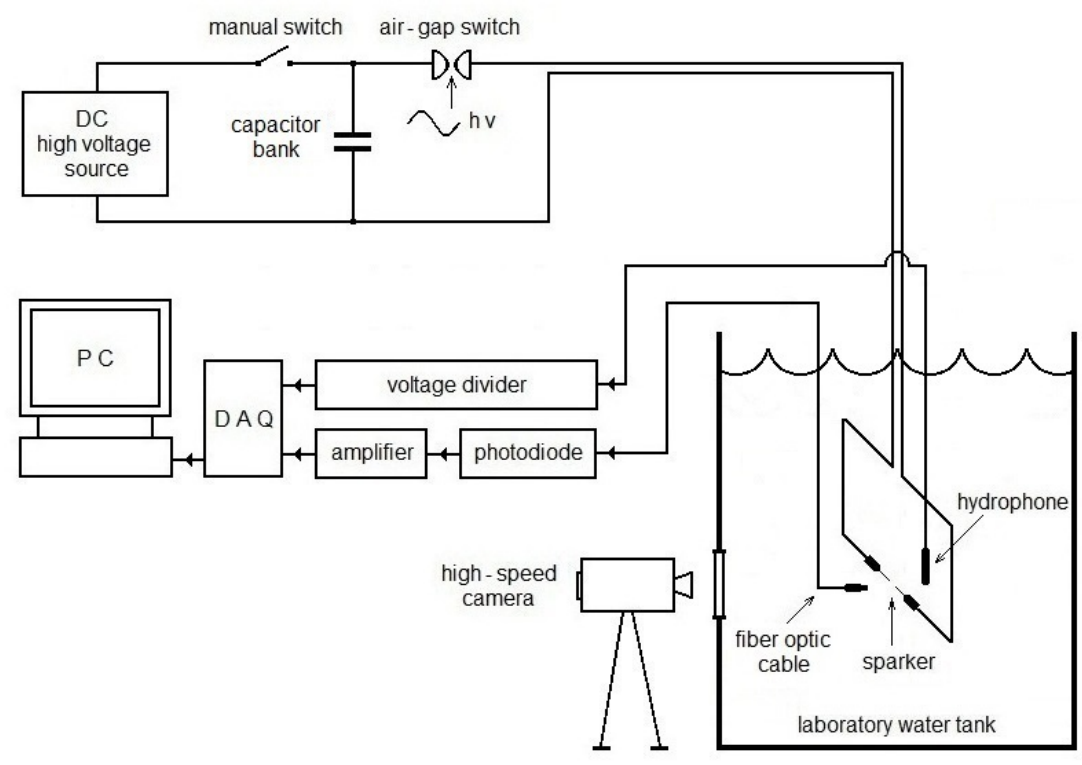

FiguRE 1. Experimental setup used to generate oscillating bubbles and to record the optical and acoustic radiation from them (abbreviations in the figure: DAQ - data acquisition board, hv - additional high voltage used to trigger the air gap).

(depth). The experiments were performed in tap water at a constant hydrostatic pressure $p_{\infty}=125 \mathrm{kPa}$, at a room temperature $\Theta_{\infty}=292 \mathrm{~K}$, and far from any boundaries. The capacitance of the capacitor bank could be varied in steps by connecting 1 to 10 capacitors in parallel. Each of these capacitors had a capacitance of $16 \mu \mathrm{F}$. The capacitors were charged from a high voltage source of $4 \mathrm{kV}$. An air-gap switch was used to trigger the discharge through the sparker. Earlier measurements 24 have shown that the current flowing through the discharge circuit has the form of a highly damped sinusoid and depending on the total bank capacity, it drops to zero in $0.3-0.7 \mathrm{~ms}$ after the liquid breakdown. A more detailed description of the experimental setup is given in an earlier work [24].

Both the spark discharge and the subsequent bubble oscillations were accompanied by an intensive optical radiation and acoustic radiation. The optical radiation was monitored by a detector, which consisted of a fiber optic cable, a photodiode, an amplifier, and an A/D converter. The input surface of the fiber optic cable was positioned in water at the same level as the sparker at a distance $r=0.2 \mathrm{~m}$ aside, pointing perpendicularly to the sparker gap and the electrodes. At the output surface of the fiber optic cable, a Hammamatsu photodiode type S2386-18L was positioned. The usable spectral range of the photodiode is $320 \mathrm{~nm}$ to $1100 \mathrm{~nm}$. An analysis of the optical spectra given in the literature showed that the maximum temperatures in spark-generated and laser-generated bubbles range from $5800 \mathrm{~K}$ to $8150 \mathrm{~K}$ [9, 14. Then, using the Wien and Planck law, it can be verified that the spectral maxima of the optic radiation are within the photodiode band-pass and that the prevailing part of the radiation is received by the detector. The load resistance of the photodiode was $75 \Omega$, so the rise time of the measured pulses is about 50 ns. A broadband amplifier $(0-10 \mathrm{MHz})$ was connected to the photodiode output terminals. The output voltage from the amplifier was recorded using a data acquisition board (National Instruments PCI 6115, 12 bit A/D converter) with a sampling frequency of $10 \mathrm{MHz}$. The presented optical data are referring to the photodiode output.

The acoustic radiation was monitored using a Reson broadband hydrophone type TC 4034. The hydrophone was positioned with the sensitive element at the same depth as the sparker. The distance between the hydrophone acoustic centre and the sparker gap was $r_{h}=0.2 \mathrm{~m}$. The output of the hydrophone was connected via a divider 10:1 to the second channel of the A/D converter.

In the experiments, a large number of almost spherical bubbles freely oscillating in a large expanse of liquid were successively generated. The sizes of these bubbles, as described by the first maximum radius $R_{\mathrm{M} 1}$, ranged from $18.5 \mathrm{~mm}$ to $56.5 \mathrm{~mm}$, and the bubble oscillation intensity, as described by the nondimensional peak pressure in the first acoustic pulse $p_{\mathrm{zp} 1}=\left(p_{\mathrm{p} 1} r_{h}\right) /\left(p_{\infty} R_{\mathrm{M} 1}\right)$ ranged from 24 to 153 [21]. Here, $p_{\mathrm{p} 1}$ is the peak pressure in the first acoustic pulse $p_{1}(t)$. The non-dimensional quantity $p_{\text {zp1 }}$ can be best interpreted by multiplying it by the hydrostatic pressure $p_{\infty}$. Then it represents the peak acoustic pressure $p_{\mathrm{p} 1}$ in the first acoustic pulse $p_{1}(t)$ measured at a distance $r_{h}=R_{\mathrm{M} 1}$. Both $R_{\mathrm{M} 1}$ and $p_{\mathrm{zp} 1}$ were determined 
in each experiment from the respective pressure record using an iterative procedure described in detail in [21. This iterative procedure is an extension of the wellknown Rayleigh's formula for the "collapse time" of a bubble having a size $R_{\mathrm{M} 1}$. The Rayleigh formula is commonly used in studies of spark and laser generated bubbles (see, e.g. 11, 9]). It has been verified experimentally many times that for bubbles oscillating sufficiently intensively, it gives satisfactory results. However, for bubbles oscillating with lower intensity, it gives less precise values. The iterative procedure is thus extending this approach to any oscillation intensity.

Prior to the measurements reported here, a limited number of high-speed camera records were taken with framing rates ranging from 2800 to 3000 frames/s. These records were used to check the shape of the generated bubbles and the photographs yielded also useful visual information on the bubble content. Examples of the photographs of the spark-generated bubbles taken by the high-speed camera at different instants of their life and the experimentally determined variations of the bubble radius $R$ with time $t$ were given in earlier works [21, 25].

\section{Results}

Let us assume that at a time $t_{0}$, the liquid breakdown initiates a spark-discharge. Thus at the instant $t_{0}$, the bubble starts growing explosively and radiating light (optical) and pressure (acoustic) waves intensively. The instant $t_{0}$ thus represents the beginning of all the physical processes considered here. The bubble wall motion is oscillatory. At a time $t_{1}$, the explosively growing spherical bubble attains its first maximum volume (a sphere of radius $R_{\mathrm{M} 1}$ ). Then the bubble starts contracting and at a time $t_{\mathrm{c} 1}$, it attains its first minimum volume (a sphere of radius $R_{\mathrm{m} 1}$ ). Then the bubble starts expanding again and at a time $t_{2}$, it attains its second maximum volume (a sphere of radius $R_{\mathrm{M} 2}$ ). After time $t_{2}$, the bubble performs several further oscillations. However, these are already out of scope of the present work. The interval $\left(t_{0}, t_{1}\right)$ represents the growth phase, the interval $\left(t_{1}, t_{\mathrm{c} 1}\right)$ the first contraction phase and the interval $\left(t_{\mathrm{c} 1}, t_{2}\right)$ the first expansion phase. The interval $\left(t_{0}, t_{\mathrm{c} 1}\right)$ represents the time of the first bubble oscillation $T_{\mathrm{o} 1}$. In this work, we shall concentrate on the processes taking place in a very short interval encompassing the final stages of the bubble contraction and the early stages of the bubble expansion. To abbreviate the description of this interval, a term "subinterval in the vicinity of the minimum bubble volume" (shortly "subinterval MBV") will be used in the following. This subinterval is centred on the instant $t_{\mathrm{c} 1}$, when the bubble is compressed to its first minimum volume and the extent of this subinterval is about $0.5 \%$ of the time of the first bubble oscillation $T_{\mathrm{o} 1}$.

As already said in Section 2, both the spark discharge and the subsequent bubble oscillations are accompanied by an intensive optical radiation and acoustic radiation. An example of an optical record, represented by the voltage $u(t)$ at the output of the optical detector, is given in Figure 2 As can be seen, the voltage $u(t)$ consists of two pulses. First, it is a pulse $u_{0}(t)$ that corresponds to the optical radiation from the bubble during the growth phase $\left(t_{0}, t_{1}\right)$. Second, it is a pulse $u_{1}(t)$ that represents the optical radiation from the bubble during the first contraction phase and the first expansion phase that is in the interval $\left(t_{1}, t_{2}\right)$. In this work, only the pulse $u_{1}(t)$ will be considered, and therefore the pulse $u_{0}(t)$ is shown clipped in Figure 2. The maximum value of the pulse $u_{1}(t)$ is denoted as $u_{\mathrm{M} 1}$ and the time of its occurrence is denoted as $t_{\mathrm{u} 1}$.

An example of an acoustic record $p(t)$ is given in Figure 3. The pressure wave has been measured at a distance from the bubble center $r_{h}=0.2 \mathrm{~m}$ and recalculated to the nominal distance $r_{n}=1 \mathrm{~m}$. As can be seen, the pressure wave also consists of several pulses. First, it is a pressure pulse $p_{0}(t)$ radiated by the bubble during the growth phase $\left(t_{0}, t_{1}\right)$. Second, it is a pressure pulse $p_{1}(t)$ radiated by the bubble during the first contraction phase and the first expansion phase that is in the interval $\left(t_{1}, t_{2}\right)$. The peak value of the pulse $p_{1}(t)$ is denoted as $p_{\mathrm{p} 1}$ and the time of its occurrence is denoted as $t_{\mathrm{p} 1}$. Further pressure pulse $p_{2}(t)$ can also be seen in Figure 3 However, this pulse will not be considered in this work.

The pressure wave propagates from the bubble wall to the hydrophone at a distance $r_{h}=0.2 \mathrm{~m}$ at the speed of sound in water $c=1482 \mathrm{~m} / \mathrm{s}$ and thus the instants $t_{0}, t_{1}, t_{\mathrm{p} 1}$, and $t_{2}$ in the pressure record are delayed by about $135 \mu \mathrm{s}$ after the instants $t_{0}, t_{1}, t_{\mathrm{c} 1}$, and $t_{2}$ defined above for the bubble wall motion. However, to simplify the discussion the propagation time of the pressure wave in water is not considered here and it will be assumed that the instants $t_{0}$ defined above for the beginning of the bubble wall motion, optical radiation, and acoustic radiation are identical, even if the hydrophone is at the distance $r_{h}$.

From the above discussion, it is evident that an accurate determination of the instants $t_{0}$ in the optical and acoustic records is crucial in this work. The instants $t_{0}$ are defined as the points in the records at which the pulses $u_{0}(t)$ and $p_{0}(t)$ start rising steeply from an undisturbed level. Small portions of the optical and acoustic pulses $u_{0}(t)$ and $p_{0}(t)$ extracted from the records at the vicinity of $t_{0}$ are displayed together in Figure 4. It can be seen that the instant $t_{0}$ can be determined relatively accurately. The precision of the determination of $t_{0}$ is given by the sampling interval $\mathrm{d} t=1 / f_{s}=0.1 \mu \mathrm{s}$.

The instants $t_{0}$ have been defined as the starting points of the recorded waves $u(t)$ and $p(t)$. In the following, the instants $t_{0}$ in both waves will be assumed to be identical, that is, the propagation time of the pressure wave will be ignored, or, which is the same, it will be assumed that the pressure wave propagates 


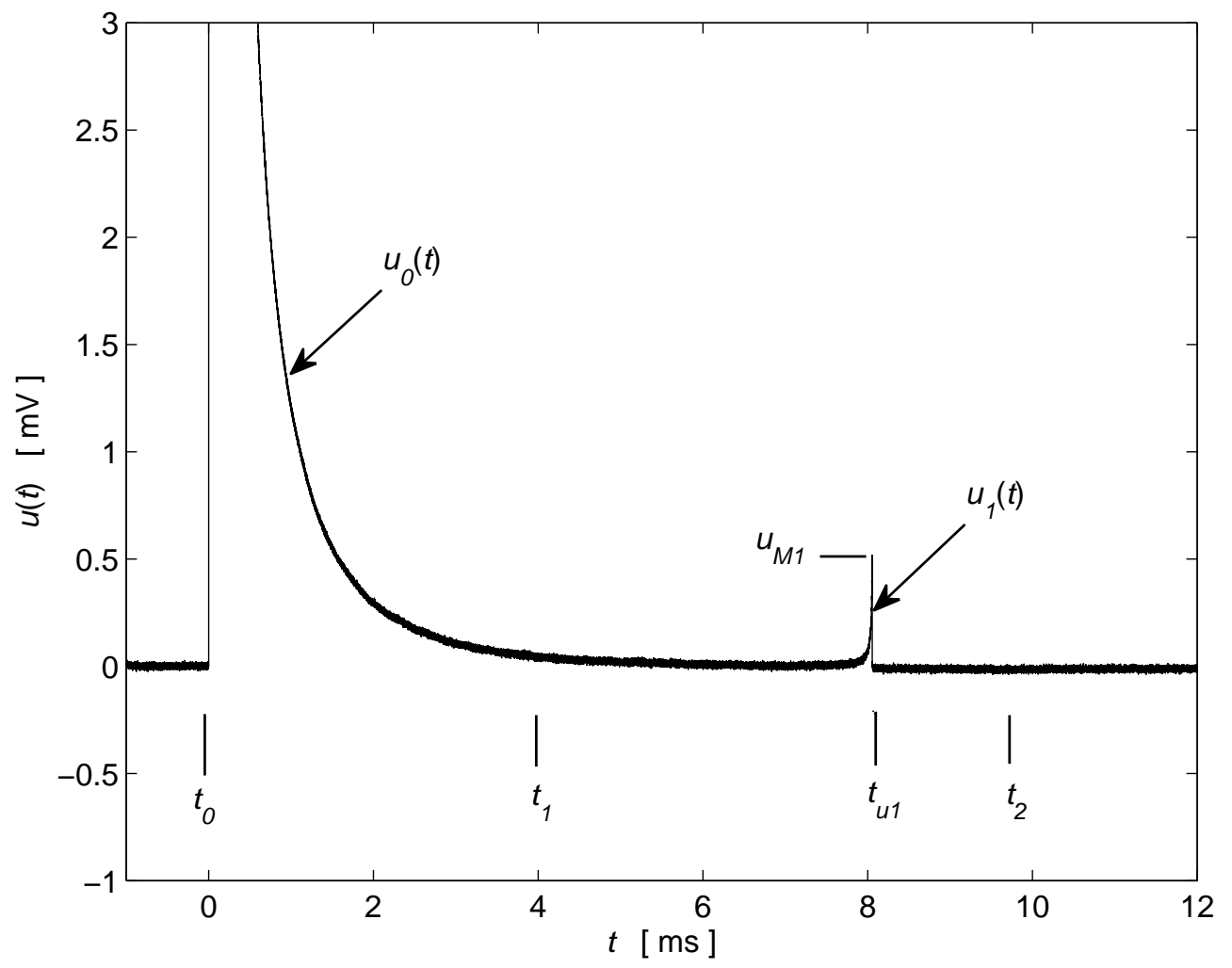

Figure 2. An example of a radiated optical wave $u(t)$. The bubble size is $R_{\mathrm{M} 1}=49 \mathrm{~mm}$, the intensity of bubble oscillation is $p_{\mathrm{zp} 1}=142.1$.

with the speed of light. Then, both waveforms can be displayed together in one figure with an identical starting point $t_{0}$ on the time axis. And in this way, both waveforms can easily be mutually compared (see also Figure 4). Because in this work, we are interested in comparison of the optical and acoustic radiations from the bubble at the subinterval MBV, only small portions of the pulses $u_{1}(t)$ and $p_{1}(t)$ extracted from the records in the vicinity of the instants $t_{\mathrm{u} 1}$ and $t_{\mathrm{p} 1}$ will be considered in the following discussion.

Examples of the pulses $u_{1}(t)$ and $p_{1}(t)$ recorded in two different experiments are shown in Figures 5 and 6. In these figures, the time origins have been set at the instants $t_{\mathrm{p} 1}$ and the sizes of the waveforms $u_{1}(t)$ have been adjusted by using arbitrary units so that the shapes of both waveforms can be compared easily. And as said above, the instants $t_{0}$ of both waves are identical on the time axis.

It can be seen in Figures 5 and 6 that the shapes of the pulses $u_{1}(t)$ and $p_{1}(t)$ differ, and that the times $t_{\mathrm{u} 1}$ and $t_{\mathrm{p} 1}$ are not identical. The difference in shapes of the pulses $u_{1}(t)$ and $p_{1}(t)$ is evidently connected with the autonomous behaviour of plasma in the bubble interior, already discussed in [25-27]. The difference in times $t_{\mathrm{u} 1}$ and $t_{\mathrm{p} 1}$ has not been discussed yet and the existence of this time difference is a surprising fact because a "reasonable" assumption is that the maxima in optical and acoustic radiations will occur at the same instant $t_{\mathrm{c} 1}$, when the bubble is contracted to the first minimum volume. Even if this has not yet been verified experimentally, it seems highly probable that the instants $t_{\mathrm{c} 1}$ and $t_{\mathrm{p} 1}$ are identical. However, this assumption then means that the maximum in the optical radiation is not firmly tied to the instant of the bubble maximum contraction $t_{\mathrm{c} 1}$, but can occur a bit earlier (Figure 5), or a bit later (Figure 6). In Figures 5 and 6 the time difference between the instants $t_{\mathrm{p} 1}$ and $t_{\mathrm{u} 1}$ has been denoted as $\delta_{1}$ and this quantity is defined by the relation $\delta_{1}=t_{\mathrm{u} 1}-t_{\mathrm{p} 1}$. As shown in Figures 5 and 6 the time difference $\delta_{1}$ can have both a positive and a negative value.

As said in Section 2, the spark-generated bubble is described by two parameters, by its size $R_{\mathrm{M} 1}$ and oscillation intensity $p_{\mathrm{zp} 1}$. It is convenient to characterise the optical pulse $u_{1}(t)$ by two parameters as well [26]. First, it is the maximum value of the pulse $u_{\mathrm{M} 1}$. Second, it is the pulse width $\Delta$ at one-half of the maximum value (that is at $u_{\mathrm{M} 1} / 2$ ). Thus defined pulse widths $\Delta$ are shown in Figures 5 and 6 In the following, the dependence of the time differences $\delta_{1}$ on these four parameters, that is on $R_{\mathrm{M} 1}, p_{\mathrm{zp} 1}, u_{\mathrm{M} 1}$ and $\Delta$, will be shown and discussed.

The variation of the time difference $\delta_{1}$ with the bubble size $R_{\mathrm{M} 1}$ determined on a larger set of experimental data is shown in Figure 7. The regression line for the mean value of the time difference $\delta_{1}$ in dependence on $R_{\mathrm{M} 1}$ is $\left\langle\delta_{1}\right\rangle=-0.2 R_{\mathrm{M} 1}+5.9$ [us, mm]. It can be seen in Figure 7 that the time difference $\delta_{1}$ is correlated with the bubble size $R_{\mathrm{M} 1}$ only very weakly and that the dispersion of the time differences $\delta_{1}$ grows with the bubble size $R_{\mathrm{M} 1}$. 


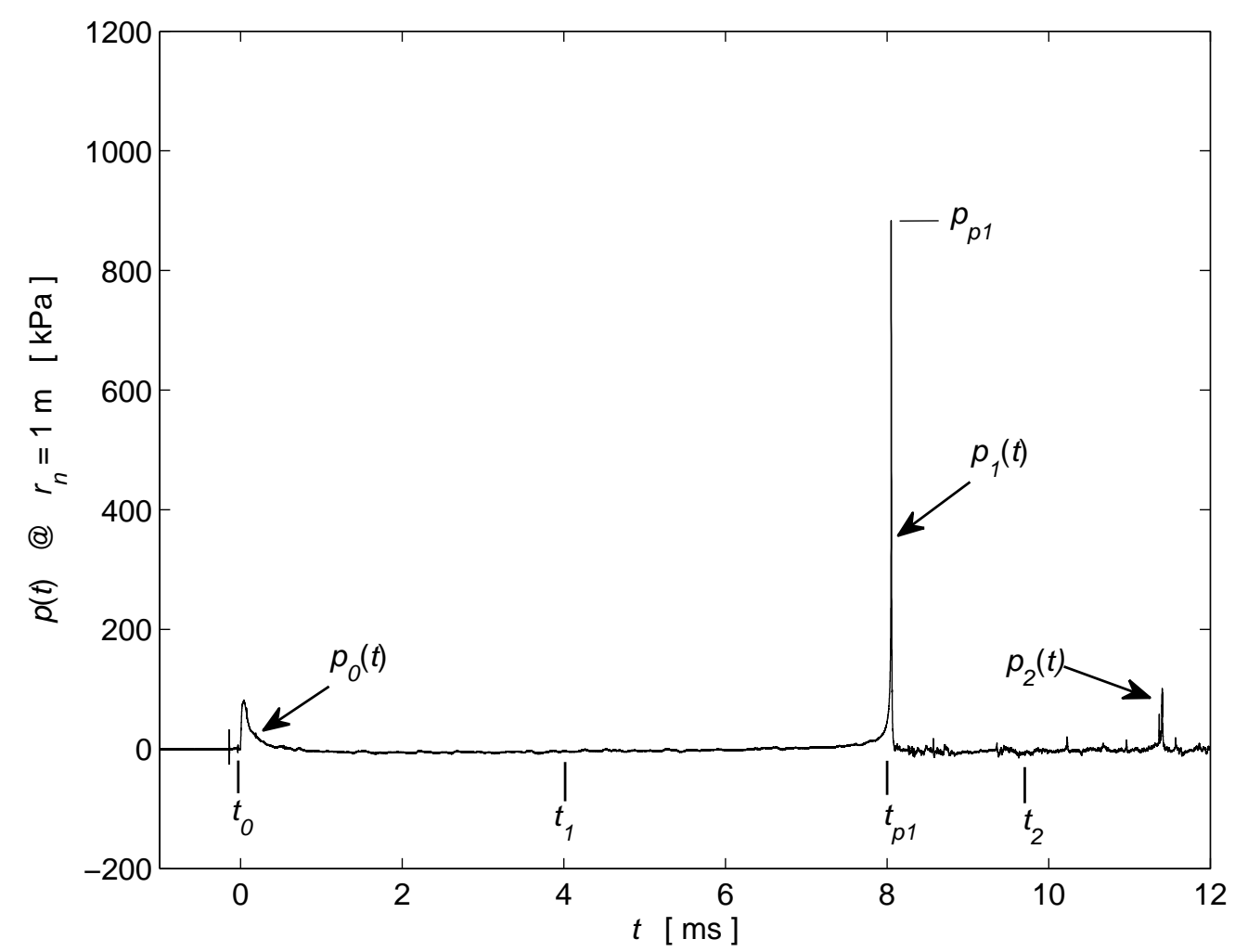

Figure 3. An example of a radiated pressure wave $p(t)$. The bubble size is $R_{\mathrm{M} 1}=49 \mathrm{~mm}$, the intensity of bubble oscillation is $p_{\mathrm{zp} 1}=142.1$.

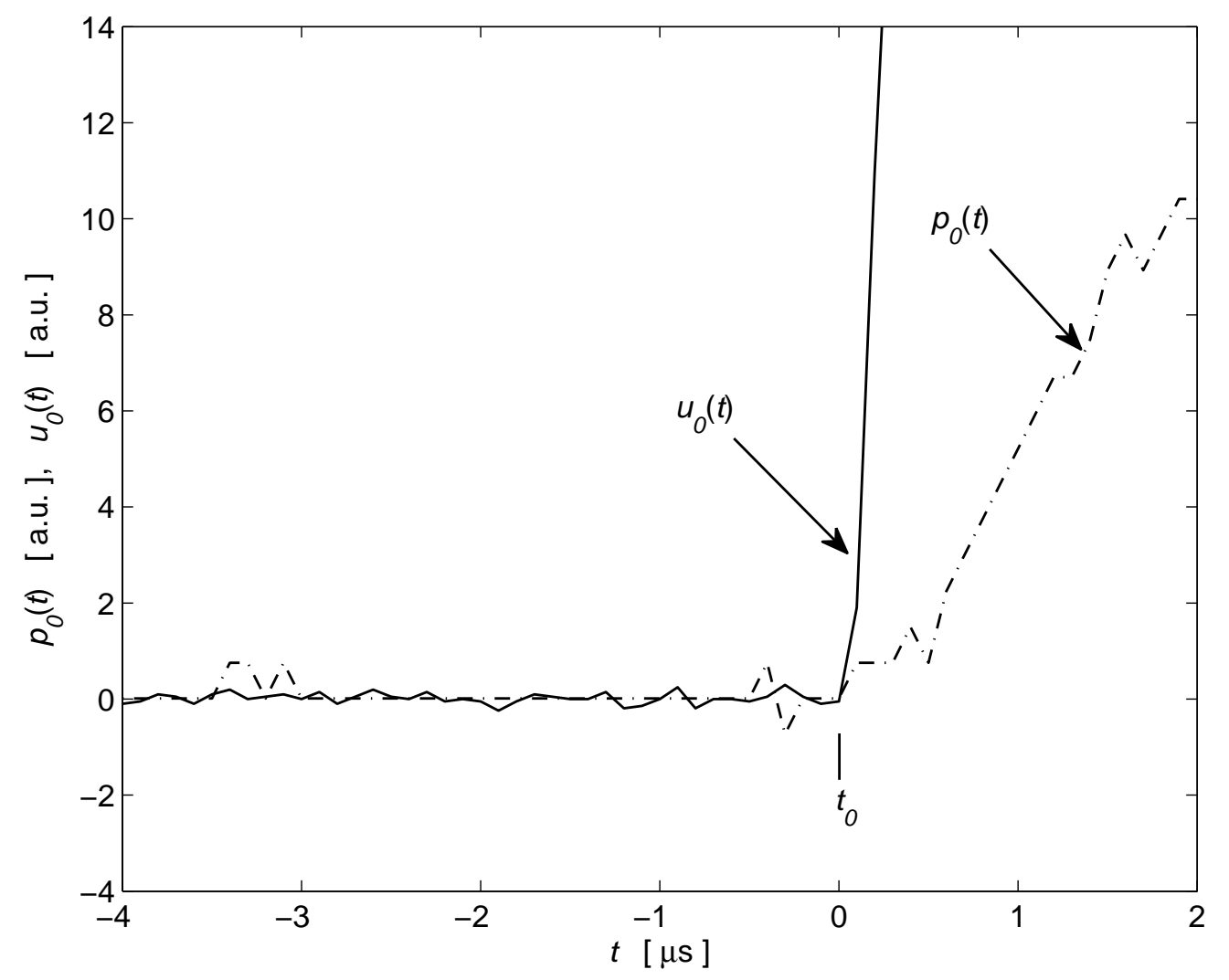

FIgURE 4. An example of voltage and pressure pulses $u_{0}(t)$ and $p_{0}(t)$ in the vicinity of the instant $t_{0}$. The bubble size is $R_{\mathrm{M} 1}=49 \mathrm{~mm}$, the intensity of bubble oscillation is $p_{\mathrm{zp} 1}=142.1$. 


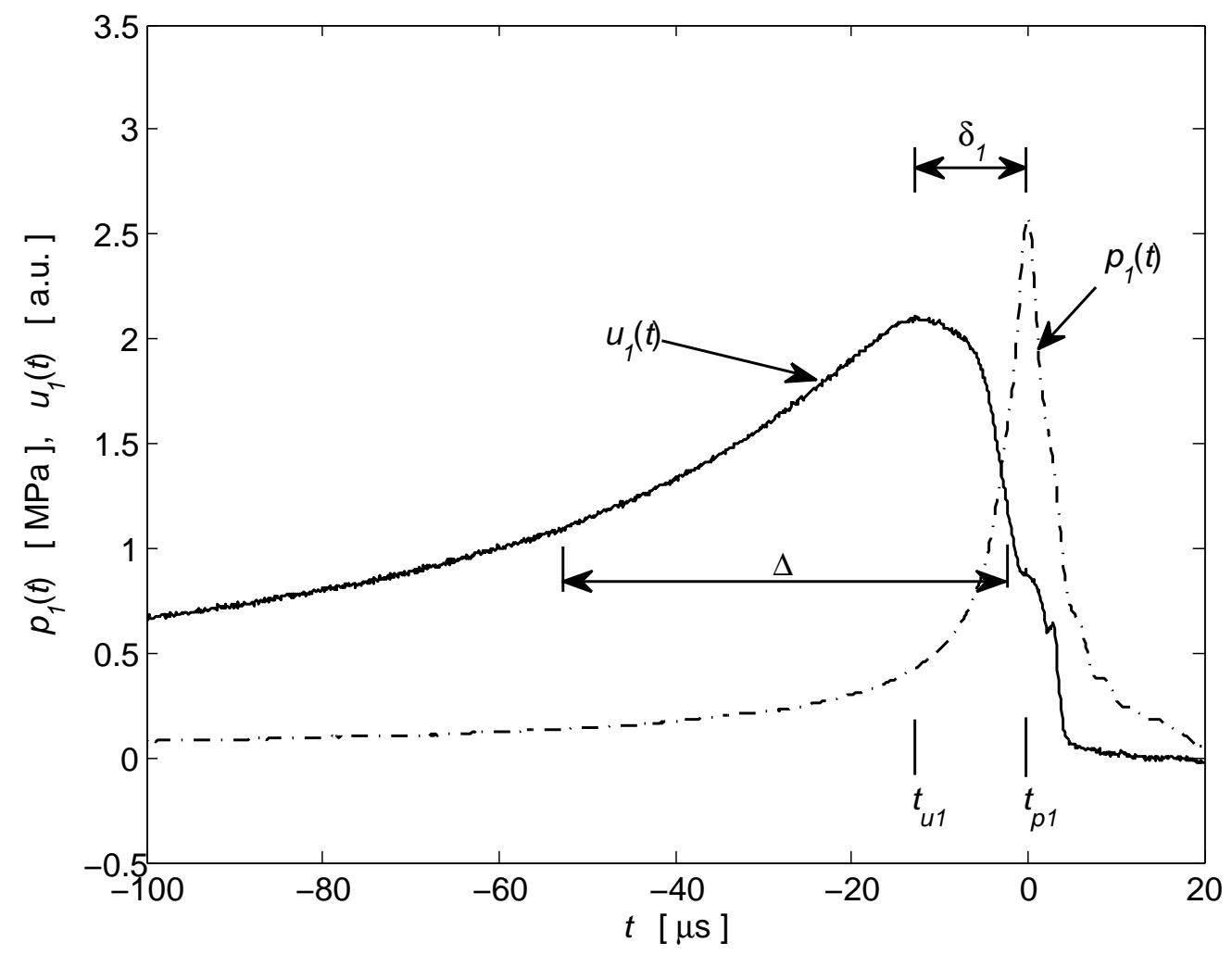

Figure 5. An example of optical and pressure waves in the vicinity of instants $t_{\mathrm{u} 1}$ and $t_{\mathrm{p} 1}$. The bubble size is $R_{\mathrm{M} 1}=38.1 \mathrm{~mm}$, the intensity of bubble oscillation is $p_{\mathrm{zp} 1}=107$, the hydrophone was at a distance $r_{h}=0.2 \mathrm{~m}$, the optical pulse width is $\Delta=57.9 \mu \mathrm{s}$, and the time difference in occurrence of the maxima in both pulses is $\delta_{1}=-12.7 \mu \mathrm{s}$.

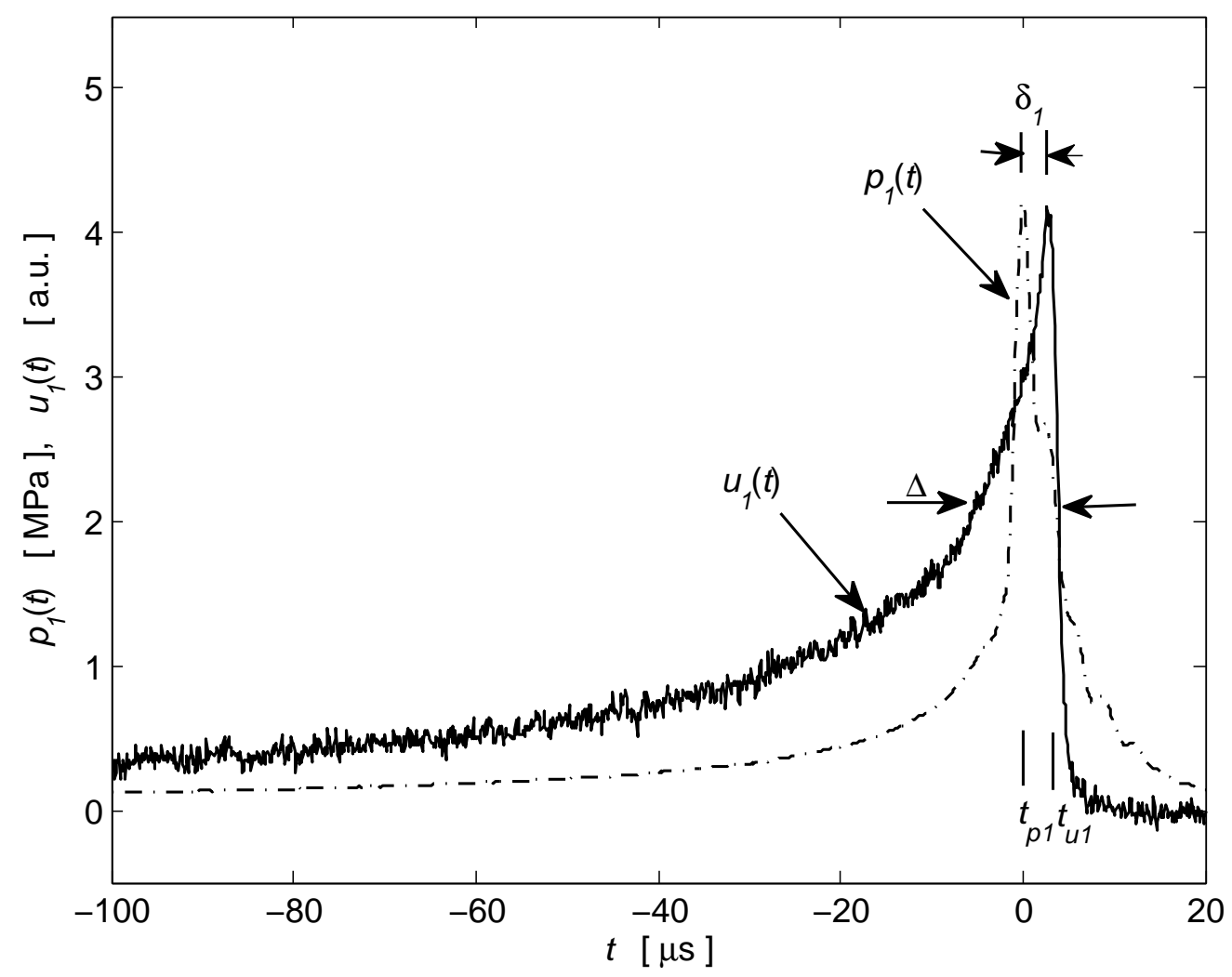

FiguRE 6. An example of optical and pressure waves in the vicinity of instants $t_{\mathrm{u} 1}$ and $t_{\mathrm{p} 1}$. The bubble size is $R_{\mathrm{M} 1}=49 \mathrm{~mm}$, the intensity of bubble oscillation is $p_{\mathrm{zp} 1}=142.1$, the hydrophone was at a distance $r_{h}=0.2 \mathrm{~m}$, the optical pulse width is $\Delta=9.4 \mu \mathrm{s}$, and the time difference in occurrence of the maxima in both pulses is $\delta_{1}=2.6 \mu \mathrm{s}$. The waveforms displayed in Figures 2, 3, 4 and 6 were recorded in the same experiment. If the records shown in Figures 2 and 3 are aligned as it is done in Figure 4 the overlapping records shown in Figure 6 are obtained. 


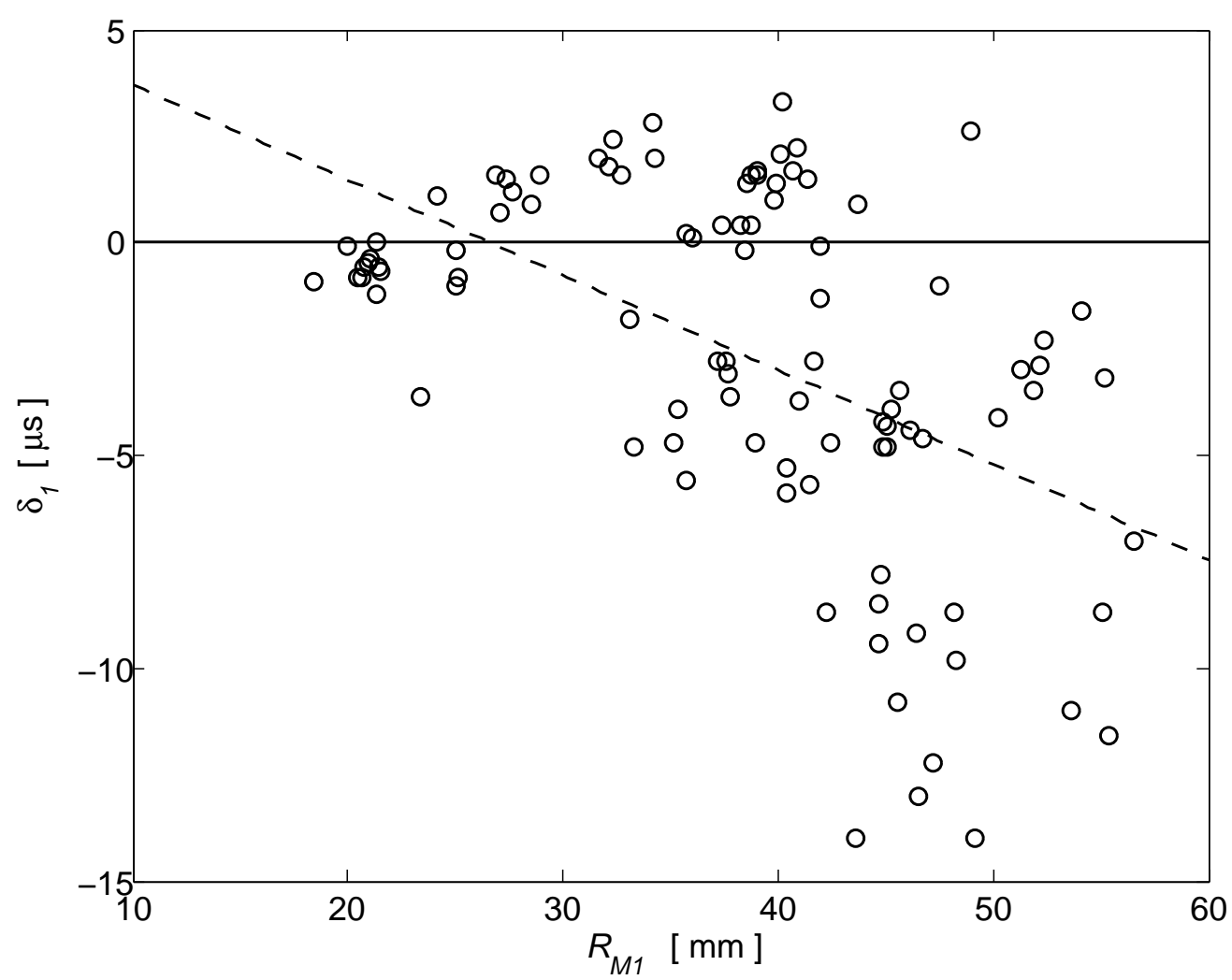

Figure 7 . The variation of the time difference $\delta_{1}$ with the bubble size $R_{\mathrm{M} 1}$.

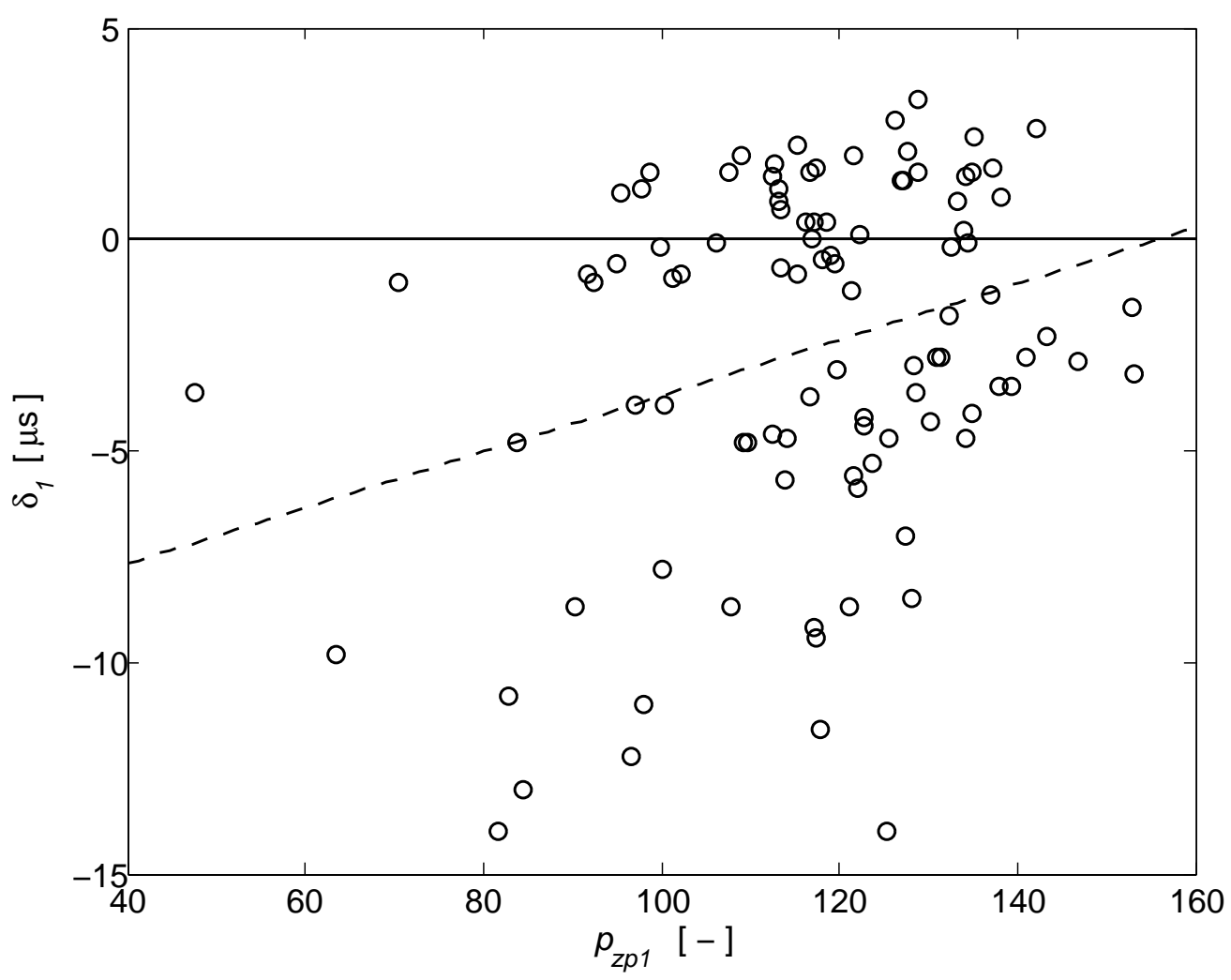

Figure 8 . The variation of the time difference $\delta_{1}$ with the bubble oscillation intensity $p_{\text {zp } 1}$. 


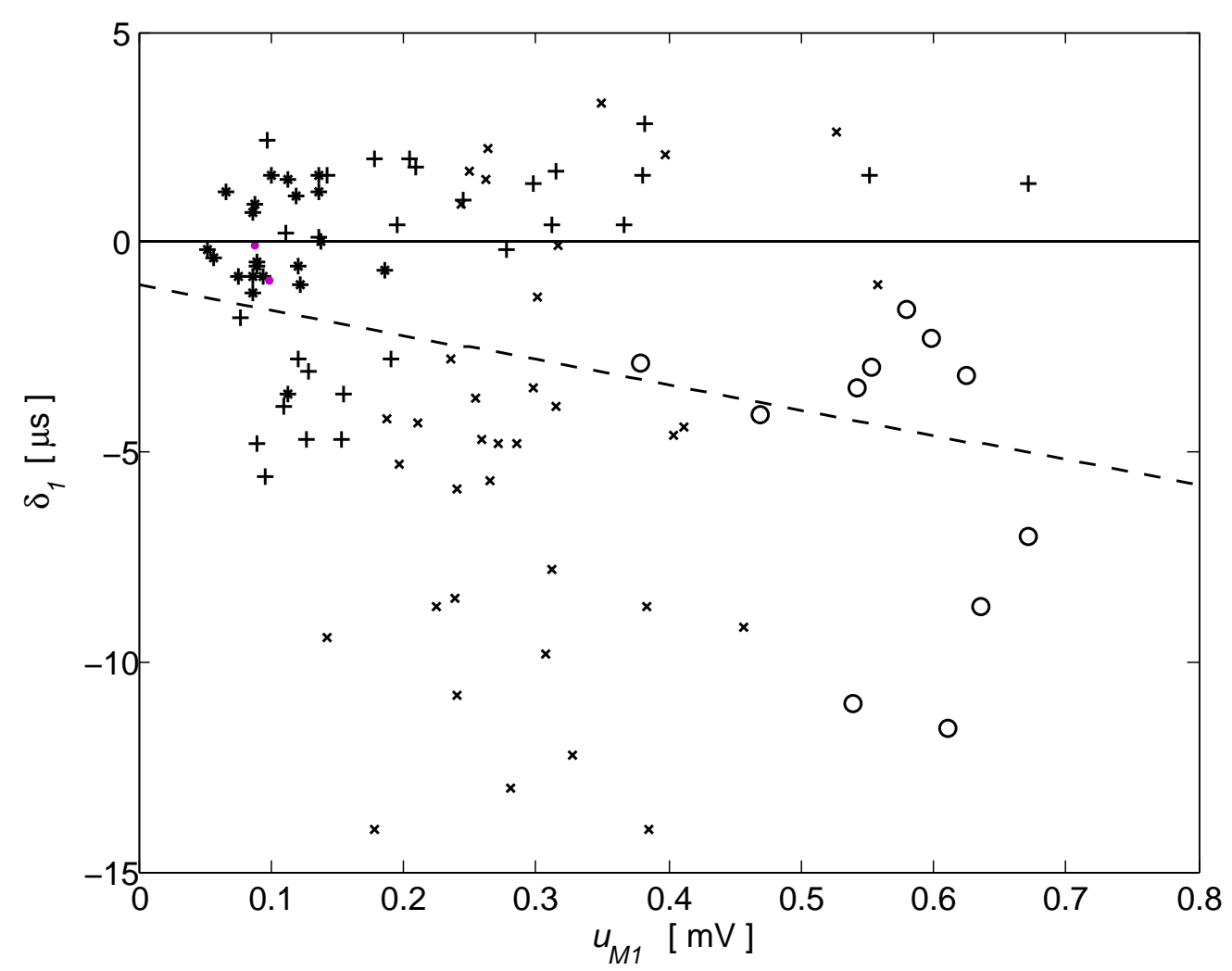

Figure 9. The variation of the time difference $\delta_{1}$ with the maximum voltage $u_{\mathrm{M} 1}$. Bubble sizes: (o) $R_{\mathrm{M} 1}>50 \mathrm{~mm}$, (×) $50 \mathrm{~mm} \geq R_{\mathrm{M} 1}>40 \mathrm{~mm},(+) 40 \mathrm{~mm} \geq R_{\mathrm{M} 1}>30 \mathrm{~mm},(*) 30 \mathrm{~mm} \geq R_{\mathrm{M} 1}>20 \mathrm{~mm},(\cdot) 20 \mathrm{~mm} \geq R_{\mathrm{M} 1}$.

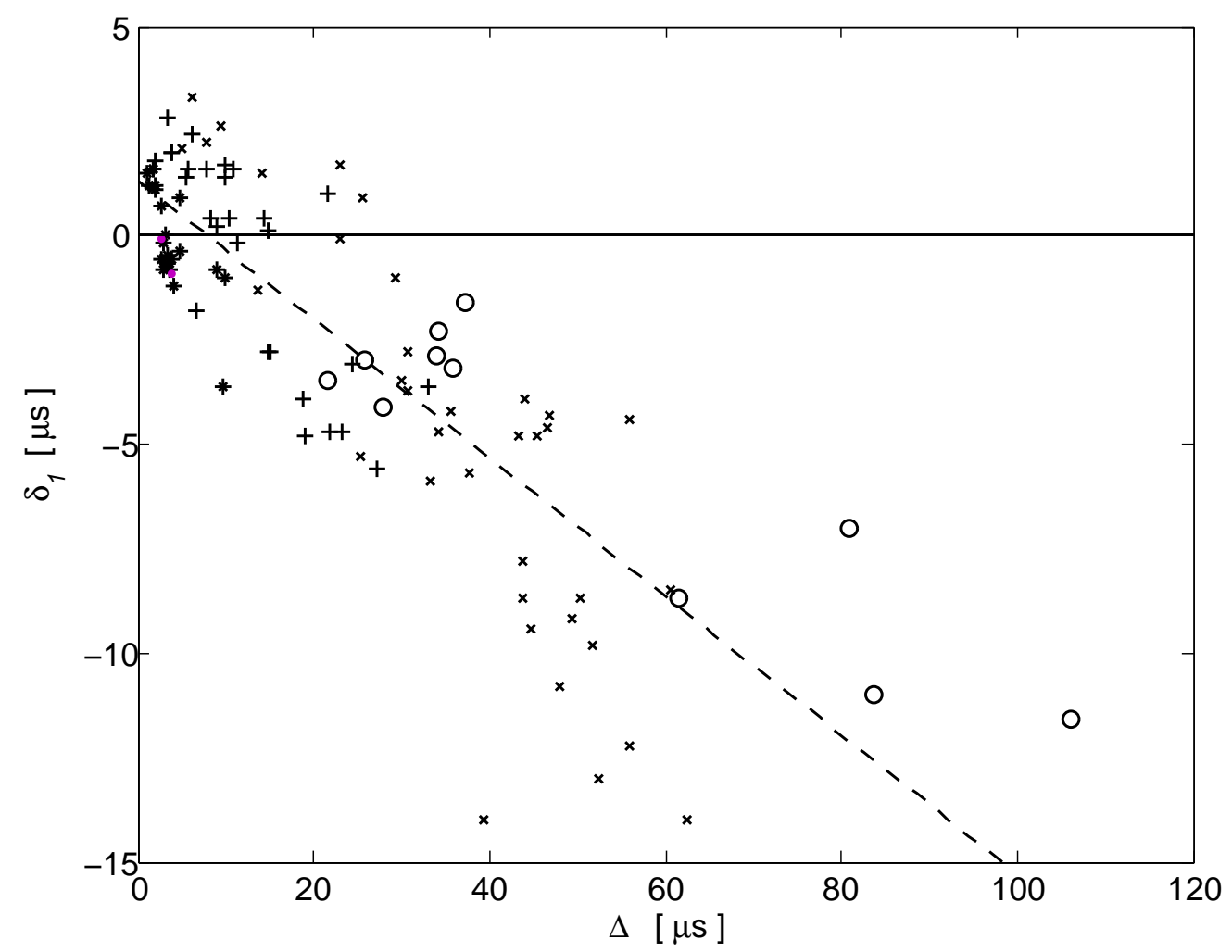

Figure 10. The variation of the time difference $\delta_{1}$ with the pulse width $\Delta$. Bubble sizes: (o) $R_{\mathrm{M} 1}>50 \mathrm{~mm},(\times)$ $50 \mathrm{~mm} \geq R_{\mathrm{M} 1}>40 \mathrm{~mm},(+) 40 \mathrm{~mm} \geq R_{\mathrm{M} 1}>30 \mathrm{~mm},(*) 30 \mathrm{~mm} \geq R_{\mathrm{M} 1}>20 \mathrm{~mm},(\cdot) 20 \mathrm{~mm} \geq R_{\mathrm{M} 1}$. 
The variation of the time difference $\delta_{1}$ with the bubble oscillation intensity $p_{\mathrm{zp} 1}$ is shown in Figure 8 The regression line for the mean value of the time difference $\delta_{1}$ in dependence on $p_{\mathrm{zp} 1}$ is $\left\langle\delta_{1}\right\rangle=0.07 p_{\mathrm{zp} 1}-10.3[\mathrm{\mu s},-$ ]. It can be seen that the time difference $\delta_{1}$ is very weakly correlated with the bubble oscillation intensity $p_{\mathrm{zp} 1}$ and that is another proof of the autonomous behaviour of plasma in the bubble interior that has already been observed earlier in works [25-27]. As discussed in [25-27], this autonomous behaviour manifests itself in the relatively large independence of the light radiation from the plasma surface on the pressure at the bubble wall.

The variation of the time difference $\delta_{1}$ with the maximum voltage in the optical pulse $u_{\mathrm{M} 1}$ (and thus with the maximum intensity of the light radiated by the bubble) is shown in Figure 9 The regression line for the mean value of the time difference $\delta_{1}$ in dependence on $u_{\mathrm{M} 1}$ is $\left\langle\delta_{1}\right\rangle=-5.9 u_{\mathrm{M} 1}-1.03[\mu \mathrm{s}, \mathrm{mV}]$. It can be seen again that the time difference $\delta_{1}$ is weakly correlated with the maximum voltage in the optical pulse $u_{\mathrm{M} 1}$. However, the dependence of the time difference $\delta_{1}$ on the bubble size $R_{\mathrm{M} 1}$, already observed in Figure 7] can also be seen in Figure 9 .

The variation of the time difference $\delta_{1}$ with the pulse width $\Delta$ is shown in Figure 10 . The regression line for the mean value of the time difference $\delta_{1}$ in dependence on $\Delta$ is $\left\langle\delta_{1}\right\rangle=-0.17 \Delta+1.3[\mu \mathrm{s}, \mu \mathrm{s}]$. It can be seen that now, the time difference $\delta_{1}$ is moderately correlated with the optical pulse width $\Delta$. For broader optical pulses $u_{1}(t)$ (that is for light flashes with larger widths $\Delta$ ), the time difference $\delta_{1}$ is negative, which means that the light flashes are radiated before the pressure pulses $p_{1}(t)$ (and thus also before the bubble contraction to $\left.R_{\mathrm{m} 1}\right)$. However, for narrower optical pulses $u_{1}(t)$ (that is, for light flashes with smaller widths $\Delta$ ), the time difference $\delta_{1}$ is positive, which means that the optical pulses are radiated later than the pressure pulses $p_{1}(t)$ (and thus are also radiated after the instant $t_{\mathrm{c} 1}$, when the bubble volume is contracted to $R_{\mathrm{m} 1}$ ). The dependence of $\delta_{1}$ on the bubble size $R_{\mathrm{M} 1}$ can also be observed. For larger bubbles, the time difference $\delta_{1}$ is predominantly negative, for smaller bubbles, the time difference $\delta_{1}$ can be both positive and negative (cf. also Figure 7).

\section{Discussion}

When comparing the instants of occurrence of the maxima in the first optical pulse and in the first acoustic pulse, it can be seen that the times $t_{\mathrm{u} 1}$ and $t_{\mathrm{p} 1}$ may differ by a few $\mu \mathrm{s}$, in some experiments, the maxima of the optical radiation are radiated earlier than the peaks in the pressure pulses (an example of this case is given in Figure 5) and in some experiments, the maxima in optical radiation are radiated later than the peaks in pressure pulses (an example of this case is given in Figure 6). As can be seen in Figures 7 , 10 . the occurrence of the optical maxima before the pressure maxima is prevailing. However, occurrence of the optical maxima after the pressure maxima can also be seen, but it is not so frequent and occurs predominantly for smaller bubbles and for more intensively oscillating bubbles. The time differences $\delta_{1}$ between these maxima are very small when compared with the times of the first bubble oscillations $T_{\mathrm{o} 1}$, the ratio $\delta_{1} / T_{\mathrm{o} 1}$ is of the order $10^{-3}$ typically. Due to this small magnitude, it is difficult to observe the time differences $\delta_{1}$ when studying smaller bubbles such as those oscillating in acoustic resonators. At present, there is no explanation for the existence of the time differences $\delta_{1}$. But their presence further confirms the earlier findings concerning the autonomous plasma behaviour in bubbles 25, 26. As shown in Figures 7 . 10 , the time difference $\delta_{1}$ may be of both positive and negative values and these values are only very weakly correlated with the bubble size $R_{\mathrm{M} 1}$, intensity of bubble oscillation $p_{\mathrm{zp} 1}$, and maximum values of the optical radiation $u_{\mathrm{M} 1}$. The time difference $\delta_{1}$ is moderately correlated only with the optical pulse widths $\Delta$. As can be seen in Figure 10, it grows with $\Delta$. For large bubbles and for large $\Delta$, the time differences $\delta_{1}$ are of negative values, and thus the maxima $u_{\mathrm{M} 1}$ always occur before the peaks $p_{\mathrm{p} 1}$. The time difference $\delta_{1}$ can be of positive values, and thus the maxima $u_{\mathrm{M} 1}$ can be delayed behind the peaks $p_{\mathrm{p} 1}$, only for small bubbles and small optical pulse widths $\Delta$.

The time difference between $t_{\mathrm{u} 1}$ and $t_{\mathrm{p} 1}$ has also been observed by Golubnichiy et al. [11], Huang et al. [13] and Zhang et al. [14]. In these experiments, the time difference $\delta_{1}$ was negative (that is, the optical pulse occurred earlier than the pressure pulse). Variation of the time difference $\delta_{1}$ with bubble parameters and optical pulse parameters has not been studied in $11,13,14$.

For the analysis carried out in this and in previous works [25, 26], the measurement of pressure waves radiated by oscillating bubbles is essential. However, in the review paper by Crum 20, there are only 2 papers mentioned in which pressure waves radiated by oscillating bubbles were recorded [20, Section VIII, Subsection L]). And even in these 2 papers, the pressure waves were not used for a more detailed analysis. Therefore, it is not surprising that in the works included in the above review, no findings similar to those presented here and in our earlier works [25, 26] were mentioned. In the review [20, altogether 40 various theoretical models trying to clarify the origins of the light emission from bubbles are also summarized [20, Section VI, Subsections A, B, C, and Section VIII, Subsection E]). An interested reader may find the full bibliographical data of these papers and short summaries of the main results given in these works in the review. The presented theories include the hot spot model, electro-hydrodynamic hypothesis, re-entrant jet impacting the opposite bubble wall, electron-neutral atom-Bremsstrahlung, protontunnelling radiation, Becquerel effect and quantum vacuum radiation, just to name some of the hypothesis 
given in the review. However, the conclusion that can be made from the review is that none of these theoretical models has been verified experimentally and none has been accepted by the research community as a definitive valid clarification of the processes taking place in bubbles that are leading to the light emission. And, unfortunately, none of these theoretical models can also explain the facts observed when studying the light radiation from spark-generated bubbles, that is, none can explain the persisting light emission during the whole first bubble oscillation, relatively autonomous behaviour of the plasma in the bubble interior and the differences in instants of the radiation of light and pressure pulses.

In view of the fact that at present, there is no suitable theoretical explanation for the observed phenomena, at the end of this Section, we would like to draw an attention to the results published by researchers studying plasmoids generated by electrical discharges in wet air 2833 . The aim of those works is to simulate the ball lightning, also known as fireballs. The electrical discharges in wet air are performed with voltages and capacitor banks roughly similar to those used in this work, i.e. the voltages are about $5 \mathrm{kV}$ and the capacitor banks have a capacitance about $1 \mathrm{mF}$. The generated plasmoids usually have an almost spherical form and live for about $0.5-1 \mathrm{~s}$. The main conclusions of these works can be summarized as follows [33] the plasmoid consists of a hot core surrounded by cool shell, possessing a translational temperature of about 600-1300 K, an electron temperature of 2000-5000 K and a rotational temperature of about $15000 \mathrm{~K}$ and is displacing air with a warm, partially ionized wateraerosol produced by the discharge. And, according to authors of work 33, this conclusion is consistent with the ball-lightning models proposed by Shevkunov $34-$ 36, where a cation - anion recombination is inhibited by many orders of magnitude by the clustering of water around ion atomic and molecular ion cores. We believe that the strange behaviour of plasma in the spark-generated bubbles can be best compared with these results. Of course, the plasmoids that are studied in 28, 33] are generated under other conditions than is the case of plasma in spark-generated bubbles. However, the research of plasmoids most likely shows the way that should be followed in studies of the light emission from spark-generated bubbles.

\section{Conclusions}

When analysing the experimental data, it was found that there is no exact coincidence in the radiation of the light flashes and pressure pulses from the sparkgenerated bubbles at the final stages of their first contraction and early stages of their first expansion (that is, in the subinterval MBV). The time difference between the maxima of the two pulses is only a few us and is, therefore, about three orders smaller than the time of the first bubble oscillation. Thus it is not easy to detect it in the case of smaller bubbles. This time difference is a further evidence of the relatively autonomous plasma behaviour that has already been observed earlier in connection with other physical processes taking place in oscillating bubbles [25 27]. Unfortunately, at the present state of knowledge of the bubble oscillations, there is no clear explanation for this phenomenon. The only physical processes that may be considered to be similar can be observed in plasmoids generated in wet air 28,33 .

\section{ACKNOWLEDGEMENTS}

This work was supported by the Ministry of Education Youth and Sports of the Czech Republic as the research project MSM 245100304. The experimental part of this work was carried out during the author's stay at the Underwater Acoustics Laboratory of the Italian Acoustics Institute, CNR, Rome, Italy. The author wishes to thank Dr. Silvano Buogo from the CNR-INSEAN Marine Technology Research Institute, Rome, Italy, for his very valuable help in preparing the experiments.

\section{REFERENCES}

[1] A. Jayaprakash, C.-T. Hsiao, G. Chahine. Numerical and experimental study of the interaction of a sparkgenerated bubble and a vertical wall. Trans. ASME J. Fluid Eng. 134, 031301, 2012. DOI:10.1115/1.4005688

[2] P.A. Dayton, J.S. Allen, K.W. Ferrara. The magnitude of radiation force on ultrasound contrast agents. J. Acoust. Soc. Am. 112, 2183-2192, 2002. DOI:10.1121/1.1509428

[3] V. Sboros. Response of contrast agents to ultrasound. Adv. Drug Deliver. Rev. 60, 1117-1136, 2008. DOI:10.1016/j.addr.2008.03.011

[4] E.P. Stride, C.C. Coussios. Cavitation and contrast: the use of bubbles in ultrasound imaging and therapy. Proc. Inst. Mech. Eng. J. Eng. Med. 224, 171-191, 2010. DOI:10.1243/09544119JEIM622

[5] T. Faez, M. Emmer, K. Kooiman, M. Versluis, A.F.W. van der Steen, N. de Jong. 20 years of ultrasound contrast agent modelling. IEEE Trans. Ultrason. Ferroelectr. Frequency Control. 60, 7-20, 2013. DOI:10.1109/TUFFC.2013.2533

[6] D.H. Thomas, M. Butler, N. Pelekasis, T. Anderson, E. Stride, V. Sboros. The acoustic signature of decaying resonant phospholipid microbubbles. Phys. Med. Biol. 58, 589-599, 2013. DOI:10.1088/0031-9155/58/3/589

[7] T. Segers, N. de Jong, M. Versluis. Uniform scattering and attenuation of acoustically sorted ultrasound contrast agents: Modeling and experiments. J. Acoust. Soc. Am. 140, 2506-2517, 2016. DOI:10.1121/1.4964270

[8] B. Ward, D.C. Emmony. Interferometric studies of the pressures developed in a liquid during infrared-laserinduced cavitation-bubble oscillation. Infrared Phys. 32, 489-515, 1991. DOI:10.1016/0020-0891(91)90138-6

[9] E.A. Brujan, D.S. Hecht, F. Lee, G.A. Williams. Properties of luminescence from laser-created bubbles in pressurized water. Phys. Rev. E. 72, 066310, 2005. DOI:10.1103/PhysRevE.72.066310 
[10] C. Frez, G.J. Diebold. Laser generation of gas bubbles: Photoacoustic and photothermal effects recorded in transient grating experiments. J. Chem. Phys. 129, 184506, 2008. DOI:10.1063/1.3003068

[11] P.I. Golubnichiy, V.M. Gromenko, A.D. Filonenko. Microsecond scanning of the optical spectra of the electrohydrodynamic sonoluminescence (in Russian). Pisma v Zh. Tekh. Fiz. 5, 568-571, 1979.

[12] Y. Huang, H. Yan, B. Wang, X. Zhang, Z. Liu, K. Yan. The electro-acoustic transition process of pulsed corona discharge in conductive water. J. Phys. D: Appl. Phys. 47, 255204, 2014. DOI: 10.1088/0022-3727/47/25/255204.

[13] Y. Huang, L. Zhang, J. Chen, X. Zhu, Z. Liu, K. Yan. Experimental observation of the luminescence flash at the collapse phase of a bubble produced by pulsed discharge in water. Appl. Phys. Lett. 107, 184104, 2015. DOI:10.1063/1.4935206

[14] L. Zhang, X. Zhu, H. Yan, Y. Huang, Z. Liu, K. Yan. Luminescence flash and temperature determination of the bubble generated by underwater pulsed discharge. Appl. Phys. Lett. 110, 034101, 2017. DOI:10.1063/1.4974452

[15] A.H. Aghdam, B.C. Khoo, V. Farhangmehr, M.T. Shervani-Tabar. Experimental study on the dynamics of an oscillating bubble in a vertical rigid tube. Exp. Therm. Fluid Sci. 60, 299-307, 2015. DOI:10.1016/j.expthermflusci.2014.09.017

[16] I. Ko, H.-Y. Kwak. Measurement of pulse width from a bubble cloud under multibubble sonoluminescence conditions. J. Phys. Soc. Japan. 79, 124401, 2010. DOI: 10.1143/JPSJ.79.124401.

[17] T.G. Leighton, M. Farhat, J.E. Field, F. Avellan. Cavitation luminescence from flow over a hydrofoil in a cavitation tunnel. J. Fluid Mech. 480, 43-60, 2003. DOI: 10.1017/S0022112003003732.

[18] M. Farhat, A. Chakravarty, J.E. Field. Luminescence from hydrodynamic cavitation. Proc. R. Soc. A. 467, 591-606, 2011. DOI:10.1098/rspa.2010.0134

[19] N.K. Bourne, J.E. Field. Shock-induced collapse and luminescence by cavities. Phil. Trans. R. Soc. Lond. A. 357, 295-311, 1999. DOI:10.1098/rsta.1999.0328

[20] L.A. Crum. Resource papers: Sonoluminescence. J. Acoust. Soc. Am. 138, 2181-2205, 2015. DOI: 10.1121/1.4929687.

[21] S. Buogo, K. Vokurka. Intensity of oscillation of spark generated bubbles. J. Sound Vib. 329, 4266-4278, 2010. DOI:10.1016/j.jsv.2010.04.030

[22] K. Vokurka, S. Buogo. Experimental study of light emission from spark generated bubbles. In: 36 . Jahrestagung für Akustik DAGA 2010, Berlin 15.-18.3.2010 (conference proceedings on CD- ROM, Deutsche Gesellschaft für Akustik, Berlin 2010, ISBN: 978-3-9808659-8-2, editors: M. Möser et al., pp. 671672), http://kfy.fp.tul.cz/katedra/zamestnanci/vokurkakarel

[23] K. Vokurka, S. Buogo. Light from oscillating bubbles - persisting mystery. In: The 80th Acoustic Seminar, Hrdoňov 4.-6.5.2010 (conference proceedings: České vysoké učení technické v Praze, Česká akustická společnost, Prague 2010, ISBN: 978-80-01-04547-3, editors M. Brothánek and R. Svobodová, pp. 65-72), http://kfy.fp.tul.cz/katedra/zamestnanci/vokurkakarel

[24] S. Buogo, J. Plocek, K. Vokurka. Efficiency of energy conversion in underwater spark discharges and associated bubble oscillations: Experimental results. Acta Acust. united with Acust. 95, 46-59, 2009. DOI:10.3813/AAA.918126

[25] K. Vokurka, Experimental determination of temperatures in spark-generated bubbles oscillating in water. Acta Polytechnica. 57, 149-158, 2017. DOI:10.14311/AP.2017.57.0149

[26] K. Vokurka, S. Buogo. Experimental study of light emitted by spark-generated bubbles in water. Eur. Phys. J. Appl. Phys. 81, 11101, 2018. DOI:10.1051/epjap/2017170332

[27] K. Vokurka, J. Plocek. Experimental study of the thermal behavior of spark-generated bubbles in water. Exp. Therm. Fluid Sci. 51, 84-93, 2013. DOI:10.1016/j.expthermflusci.2013.07.004

[28] A.I. Egorov, S.I. Stepanov. Long-lived plasmoids produced in humid air as analogues of ball lightning. Tech. Phys. 47, 1584-1586, 2002. DOI:10.1134/1.1529952

[29] A.I. Egorov, S.I. Stepanov, G.D. Shabanov. Laboratory demonstration of ball lightning. PhysicsUspekhi. 47, 99-101, 2004. DOI:10.1070/PU2004v047n01ABEH001691

[30] Y. Sakawa, K. Sugiyama, T. Tanabe, R. More. Fireball generation in a water discharge. Plasma and Fusion Res.: Rap. Commun. 1, 039-1 - 039-2, 2006. DOI:10.1585/pfr.1.039

[31] A.I. Egorov, S.I. Stepanov. Properties of short-living ball lightning produced in the laboratory. Tech. Phys. 53, 688-692, 2008. DOI:10.1134/S1063784208060029

[32] A. Versteegh, K. Behringer, U. Fantz, G. Fussmann, B. Jüttner, S. Noack. Long-living plasmoids from an atmospheric water discharge. In: 28th Int. Conf. on Phenomenon in Ionized Gases, Prague 2007, Plasma Sources Sci. Technol. 17, 024014, 2008. DOI:10.1088/0963-0252/17/024014

[33] D.M. Friday, P.B. Broughton, T.A. Lee, G.A. Schutz, J.N. Betz. Further insight into the nature of balllightning-like atmospheric pressure plasmoids. J. Phys. Chem. A. 117, 9931-9940, 2013. DOI:10.1021/jp400001y

[34] S.V. Shevkunov. Cluster mechanism of the energy accumulation in a ball electric discharge. Dokl. Phys. 46, 467-472, 2001. DOI:10.1134/1.1390398

[35] S.V. Shevkunov. Scattering of centimeters radiowaves in a gas ionized by radioactive radiation: cluster plasma formation. J. Exp. Theor. Phys. 92, 420-440, 2001. DOI:10.1134/1.1364740

[36] S.V. Shevkunov. A high energy barrier to charge recombination in ionized water vapor. High Energy Chem. 43, 341-349, 2009. DOI:10.1134/S0018143909050026 